\title{
Quantitative assessment of pulmonary function in lymphangioleiomyomatosis patients using high-resolution computed tomography and pulmonary function tests
}

\author{
Zhiwen $\mathrm{Ni}^{1}$, Thomas S. C. $\mathrm{Ng}^{2}$, Jie Liu ${ }^{3}$, Suidan Huang ${ }^{1}$, Xiaoling $\mathrm{Li}^{1}$, Xiaoyin $\mathrm{Xu}^{2}$, Huai Chen ${ }^{1}$ \\ ${ }^{1}$ Department of Radiology, First Affiliated Hospital of Guangzhou Medical University, Guangzhou, China; ${ }^{2}$ Department of Radiology, Brigham \\ and Women's Hospital, Harvard Medical School, Boston, MA, USA; ${ }^{3}$ Department of Respiratory, First Affiliated Hospital of Guangzhou Medical \\ University, Guangzhou, China \\ Contributions: (I) Conception and design: H Chen, Z Ni; (II) Administrative support: H Chen; (III) Provision of study materials or patients: H Chen, \\ TSC Ng, J Liu, S Huang, X Li; (IV) Collection and assembly of data: H Chen, S Huang, Z Ni, X Xu; (V) Data analysis and interpretation: J Liu; (VI) \\ Manuscript writing: All authors; (VII) Final approval of manuscript: All authors. \\ Correspondence to: Huai Chen. Department of Radiology, First Affiliated Hospital of Guangzhou Medical University, Guangzhou 510120, China. \\ Email: chenhuai1977@163.com.
}

\begin{abstract}
Background: To explore the feasibility of using quantitative high-resolution computed tomography (HRCT) to evaluate pulmonary function in patients with pulmonary lymphangioleiomyomatosis (PLAM).

Methods: Pulmonary function tests (PFTs) were performed in 30 patients with pathologically confirmed PLAM with the use of HRCT. These results were correlated with quantitative HRCT in 21 patients.

Results: There were significant correlations between the HRCT parameters for lung function and PFT parameters. Among these parameters, emphysema volume (EV), pulmonary volume with a pixel index less than the trigger threshold (-950 HU) to account for a proportion of total lung volume [PI-950 (\%)] and forced expiratory volume in 1 second/forced vital capacity [FEV1/FVC (\%)] had the strongest correlations, reaching values between -0.71 and -0.68 . HRCT lung function might therefore also be helpful for predicting changes in lung function before and after treatment.

Conclusions: HRCT is helpful for the assessment of pulmonary function in PLAM patients and can assist in the clinical evaluation of lung function and treatment response in patients with this disease.
\end{abstract}

Keywords: Lymphangioleiomyomatosis; computed tomography; pulmonary function tests (PFTs); chest

Submitted Mar 02, 2020. Accepted for publication Sep 28, 2020.

doi: $10.21037 /$ jtd-20-1104

View this article at: http://dx.doi.org/10.21037/jtd-20-1104

\section{Introduction}

Pulmonary lymphangioleiomyomatosis (PLAM) is a rare disease whose pathophysiology is unclear (1). Studies have shown that the disease mainly occurs in women of childbearing age but can also be found in a small number of men with tuberous sclerosis (2). Recent studies have also shown that elevated serum vascular endothelial growth factor D (VEGF-D) can be observed in PLAM patients. Specifically, the indication of a VEGF-D index greater than $800 \mathrm{pg} / \mathrm{mL}$ with high-resolution computed tomography (HRCT) can improve the accuracy of diagnosing PLAM. The
PLAM detection rate has improved significantly in recent years due to the widespread use of HRCT and growing awareness of the disease. However, PLAM management remains challenging (3-5). The availability of new drugs and treatment methods has resulted in a high 10-year survival rate for patients with PLAM, but assessments of PLAM severity (i.e., lung function) and treatment responses need to be optimized. Given the toll that advanced stage PLAM with deterioration of lung function has on patients, their families and society at-large (6), early diagnosis and intervention are vitally important, even in asymptomatic 
patients.

While pulmonary function tests (PFTs) remain the gold standard for assessing lung function, PFTs can often be insensitive to early functional changes and can result in pneumothorax in patients with PLAM. Furthermore, PFTs do not provide anatomic information, such as the presence of pulmonary lesions, which can support the diagnosis of systemic lymphangioleiomyomatosis. Thus, a noninvasive tool that can provide both anatomic and functional information will be highly desired for the diagnosis and management of PLAM. In this study, we evaluated the feasibility of assessing pulmonary function in patients with PLAM using quantitative HRCT and compared HRCT to standard PFT assessments to identify imaging markers of pulmonary function.

\section{Methods}

\section{Population and ethics}

A total of 30 patients who were pathologically diagnosed with PLAM and had clinical symptoms including shortness of breath [17/30], cough [13/30], expectoration [5/30], chest distress [2/30], chest pain [1/30] and dyspnea [2/30] were investigated in this study. No other clinical symptoms and diseases such as asthma, haemoptysis, pulmonary hypertension, hypertension, diabetes, coronary heart disease, etc., were observed. All 30 patients were females and none had a history of smoking. Among the 30 patients, 15 cases, with an average age of $40.67 \pm 7.49$, had pulmonary involvement only, and the other 15 cases, with an average age of $33.80 \pm 7.78$, had both pulmonary and extrapulmonary involvement. The pulmonary lesions seen on CT in these patients included pulmonary cysts [30/30], ground-glass opacity $(10 \%, 3 / 30)$, consolidation shadow $(13 \%, 4 / 30)$, fibrosis $(67 \%, 20 / 30)$, interlobular septal thickening $(27 \%$, $8 / 30)$, pneumothorax $(47 \%, 14 / 30)$, pleural effusion $(17 \%$, $5 / 30)$ and pleural thickening $(40 \%, 12 / 30)$. No patients had chylothorax. The extrapulmonary lesions included hepatic hamartoma $(20 \%, 3 / 15)$, renal hamartoma $(53 \%, 8 / 15)$, splenic haemolymphangioma $(13 \%, 2 / 15)$, retroperitoneal lymphangiomas $(27 \%, 4 / 15)$, retroperitoneal lymph node enlargement $(20 \%, 3 / 15)$, pelvic cyst $(13 \%, 2 / 15)$ and cerebral tuberous sclerosis $(13 \%, 2 / 15)$. All patients underwent chest HRCT examinations during the inspiratory and expiratory phases, PFTs, and other clinically relevant tests. All 14 patients with pneumothorax had oxygen inhalation. Bed rest and oxygen inhalation was provided to only those who displayed a few instances of pneumothorax, to allow the gas to absorb automatically; however, closed thoracic drainage was the main treatment method to those had many instances of pneumothorax. No patients had pleurodesis treatment. Symptomatic treatment was performed on those without pneumothorax, for example, anti-inflammation, sputum aspiration and relieving cough, but no patients underwent treatment with bronchodilatators. After treatment, 21 (70\%, 21/30) patients were followed up for 3- to 6-month intervals. Patients underwent PFTs and HRCT PFTs before and after diagnosis. During the same period, 20 healthy people with an average age of $39.24 \pm 10.01$ were recruited as the control group and underwent bi-phasic respiratory HRCT examinations. Since the patient group was all female, the selected control group was also all women and had similar body mass index (BMI) indices.

The study was conducted in accordance with the Declaration of Helsinki (as revised in 2013). The study was approved by the institutional ethical committee of the First Affiliated Hospital of Guangzhou Medical University (Medical research ethical review 2019, No. K47), with informed consent waived due to the retrospective nature of the study.

\section{HRCT imaging}

All HRCT examinations were performed using a 16-detector row Toshiba Aquilion CT scanner that was calibrated each day prior to imaging. Before imaging, all subjects were trained to perform breath holds for both deep inspiration and expiration. Each subject was trained in breath holding up to three times and inclusion in the study was based on her ability to perform the above regimen. All subjects were scanned in the supine position with their hands on their heads and without the use of contrast agents. The imaging field of view extended from the lung apices to the diaphragm. The entire lung was imaged during a single breath hold for both the inspiratory and expiratory phases for all subjects without significant respiratory artefacts. The scanning parameters were as follows: tube voltage, $120 \mathrm{kV}$; tube current, $60 \mathrm{~mA}$; rotation time, 0.5 second; slice thickness, $1.0 \mathrm{~mm}$; acquisition matrix, $512 \times 512$; and interval, $1.0 \mathrm{~mm}$. The reconstruction layer thickness and interval and reconstruction kernel were $1.0 \mathrm{~mm}, 1.0 \mathrm{~mm}$ and $\mathrm{Fc} 01$, respectively. 
Table 1 Results of biological characteristics of healthy controls and PLAM patients

\begin{tabular}{lccc}
\hline Project & The healthy group $(\mathrm{n}=20)$ & PLAM patients $(\mathrm{n}=30)$ & $\mathrm{P}$ value \\
\hline Age (years) & $39.24 \pm 10.01$ & $37.23 \pm 8.28$ & 0.444 \\
Sex (male/female) & $0 / 20$ & $0 / 30$ & 1.000 \\
BMl $\left(\mathrm{kg} / \mathrm{m}^{2}\right)$ & $21.58 \pm 3.12$ & $20.52 \pm 3.05$ & 0.240 \\
\hline
\end{tabular}

PLAM, pulmonary lymphangioleiomyomatosis; BMI, body mass index.

\section{PFT measurements}

All subjects underwent PFTs one week after each HRCT examination using Cosmed Quark PFT (Cosmed, Rome, Italy) and SensorMedics Vmax 229 (SensorMedics Co., Yorba Linda, CA, USA). The measurements made were set to meet the ATS/ERS instrument quality control standards. We measured the total lung capacity (TLC), residual volume (RV), RV/TLC (\%), forced expiratory volume in 1 second/forced vital capacity [FEV1/FVC (\%)], FEV1 (\% pred), FVC (\% pred), peak expiratory flow (PEF) (\% pred), maximum expiratory flow (MEF) (\% pred), forced expiratory flow (FEF) $25 \%$ (\% pred), FEF50\% (\% pred), FEV1 (\% pred) after treatment, and FEV1/FVC (\%) after treatment.

\section{Quantitative analysis of HRCT lung function}

We used fully automatic software (LungCAD_SVM2.0.1) to quantitatively analyse lung function. The original DICOM data collected from the inspiratory and expiratory phases were transmitted to a designated computer. We used the software to automatically segment the thorax into the lung parenchyma, mediastinum soft tissues, trachea, great vessels, and chest wall. The segmented images were then used to automatically calculate the relevant HRCT metrics of lung function. The parameters recorded in this experiment were as follows: (I) inspiratory phase: mean lung density (MLD), lung volume (LV), emphysema volume $(\mathrm{EV})$, and pulmonary volume with a pixel index less than the trigger threshold $(-950 \mathrm{HU})$ to account for a proportion of total LV [PI-950 (\%)] and (II) expiratory phase: MLD, LV, $\mathrm{EV}$, and pulmonary volume with a pixel index less than the trigger threshold $(-950 \mathrm{HU})$ to account for a proportion of total LV [PI-950 (\%)].

\section{Statistical analysis}

All data are presented as the mean \pm standard deviation. When comparing the two groups, if the data were determined to have a normal distribution with equal variations, we used a $t$-test to compare the measurements. Otherwise, we used the Kruskal-Wallis test with a Bonferroni correction. For data that were subject to frequency distribution analysis, if the number of theoretical frequencies $T<5$ was less than one-fifth of the total number of classes, a chi-square test was used. When the number of $\mathrm{T}<5$ was greater than one-fifth of the total number of classes, Fisher's exact test was used. Correlation analysis was conducted using the Pearson test for normally distributed variables and the Spearman test for non-normally distributed variables. We considered $\mathrm{P}<0.05$ to be statistically significant.

\section{Results}

We recruited 30 patients with clinically diagnosed PLAM and 20 healthy people as the control group. No significant differences in age, sex or BMI were observed between the patients with PLAM and the healthy controls (Table 1). The PFT parameters of the patients were analysed and are detailed in Table 2.

We compared the HRCT-derived lung function parameters between the patients and healthy controls (Table 3). This comparison showed statistically significant differences in the inspiratory LV, PI-950 (\%), and the expiratory MLD between the two groups $(\mathrm{P}<0.05)$. In the inspiratory phase, the $\mathrm{LV}$ of the patients was less than that of the control group, while the PI-950 (\%) was greater for patients than for controls. In the expiratory phase, the MLD of PLAM patients was less than that of the control group, while the PI-950 (\%) was greater for patients than for controls.

We also correlated the HRCT-derived lung function parameters and PFT parameters in the PLAM patients (Table 4). In the inspiratory phase, LV showed good correlations with FEV1/FVC (\%), TLC (\% pred), TLC, and $\mathrm{FVC} \%$ (correlation coefficients were 0.3997, 0.3950, 0.4934 , and 0.3672 , respectively, $\mathrm{P}<0.05)$. EV showed good correlations with FEV1/FVC (\%), PEF (\% pred), 
FEF25-75\% (\% pred), MEF50\% (\% pred), and FVC\% (correlation coefficients were $0.6803,0.4855,0.5427$, 0.5595 , and 0.6373 , respectively, $\mathrm{P}<0.05$ ) (Figure 1A). MLD showed good correlations with FEV1/FVC (\%) and

Table 2 Clinical pulmonary function parameters in patients with PLAM

\begin{tabular}{lc}
\hline PFTs & PLAM $(\mathrm{n}=30)$ \\
\hline FEV1 & $1.69 \pm 0.68$ \\
FVC & $2.52 \pm 0.72$ \\
FEV1\%/pred & $62.47 \pm 24.75$ \\
TLC (L) & $4.23 \pm 0.72$ \\
RV (L) & $1.65 \pm 0.61$ \\
RV/TLC & $0.39 \pm 0.12$ \\
TLC - RV (L) & $2.58 \pm 0.67$ \\
FEV1/FVC (\%) & $64.74 \pm 18.19$ \\
FVC (\% pred) & $80.97 \pm 23.00$ \\
PEF (\% pred) & $67.42 \pm 24.65$ \\
FEF25-75\% (\% pred) & $33.22 \pm 21.14$ \\
MEF50\% (\% pred) & $34.33 \pm 20.90$ \\
MEF25\% (\% pred) & $32.12 \pm 22.90$ \\
RV (\% pred) & $106.59 \pm 38.29$ \\
TLC (\% pred) & $90.25 \pm 16.58$ \\
\hline PLAM. pulmonary &
\end{tabular}

PLAM, pulmonary lymphangioleiomyomatosis; PFTs, pulmonary function tests; FEV1, forced expiratory volume in 1 second; FVC, forced vital capacity; TLC, total lung capacity; RV, residual volume; PEF, peak expiratory flow; FEF, forced expiratory flow; MEF, maximum expiratory flow.
FVC\% (correlation coefficients were 0.4257 and 0.3931 , respectively, $\mathrm{P}<0.05)$. PI-950 (\%) showed good negative correlations with FEV1/FVC (\%), PEF (\% pred), FEF25$75 \%$ (\% pred), MEF50\% (\% pred), and MEF25\% (\% pred) (correlation coefficients were $-0.7106,-0.5371,-0.5639$, -0.5809 , and -0.4755 , respectively, $\mathrm{P}<0.05$ ) (Figure $1 B$ ).

In the expiratory phase, $\mathrm{LV}$ showed good correlations with FEV1/FVC (\%), PEF (\% pred), RV, and RV/TLC (correlation coefficients were $-0.4314,-0.5037,0.4415$, and 0.4269 , respectively, $\mathrm{P}<0.05)$. EV showed good correlations with FEV1/FVC (\%), PEF (\% pred), FEF25-75\% (\% pred), and FEV1 (correlation coefficients were $-0.5586,-0.5975$, -0.4053 , and -0.5100 , respectively, $\mathrm{P}<0.05$ ) (Figure $1 C$ ). MLD showed good correlations with FEV1/FVC (\%), FVC (\% pred), PEF (\% pred), FEV1, and FVC (correlation coefficients were $0.4641,0.4757,0.5643,0.5344$, and 0.4867 , respectively, $\mathrm{P}<0.05$ ). PI-950 (\%) showed good negative correlations with FEV1/FVC (\%), PEF (\% pred), RV/TLC, FEV1, FVC\%, and FEV1\%/pred (correlation coefficients were $-0.5871,-0.6230,0.5046,0.5344,-0.5309$, and -0.5268 , respectively, $\mathrm{P}<0.05$ ) (Figure $1 D$ ).

In total, 21/30 PLAM patients were followed up after clinical treatment for 3- to 6-month intervals. These patients underwent PFTs and HRCT before and after treatment. We found no significant differences in the PFT parameters before and after treatment (Table 5). The HRCT-derived lung function parameters before and after treatment were also analysed (Table 6). Interestingly, we observed that there were statistically significant differences in the mean LV before and after treatment during both the inspiratory and expiratory phases. The inspiratory mean $\mathrm{LV}$ after treatment was greater than that before treatment.

Table 3 Statistical analysis of HRCT pulmonary function in PLAM patients and healthy controls

\begin{tabular}{lccc}
\hline HRCT lung function & PLAM $(\mathrm{n}=30)$ & Healthy $(\mathrm{n}=20)$ & P value \\
\hline Expiratory phase & & & $<0.001$ \\
LV $(\mathrm{mL})$ & $3,500.65 \pm 944.54$ & $4,648.61 \pm 112.34$ & 0.077 \\
MLD $(\mathrm{Hu})$ & $-862.91 \pm 43.43$ & $-841.72 \pm 35.71$ & $<0.001$ \\
PI-950 $(\%)$ & $20.21 \pm 15.40$ & $1.62 \pm 1.70$ & 0.331 \\
Inspiratory phase & & & $<0.001$ \\
LV $(\mathrm{mL})$ & $1,887.58 \pm 970.25$ & $2,099.12 \pm 549.13$ & $<0.001$ \\
MLD $(\mathrm{Hu})$ & $-759.85 \pm 76.97$ & $-649.76 \pm 62.23$ & $0.09 \pm 0.45$ \\
PI-950 $(\%)$ & $9.56 \pm 12.72$ & 0.001 & \\
\hline
\end{tabular}

HRCT, high-resolution computed tomography; PLAM, pulmonary lymphangioleiomyomatosis; LV, lung volume; MLD, mean lung density. 


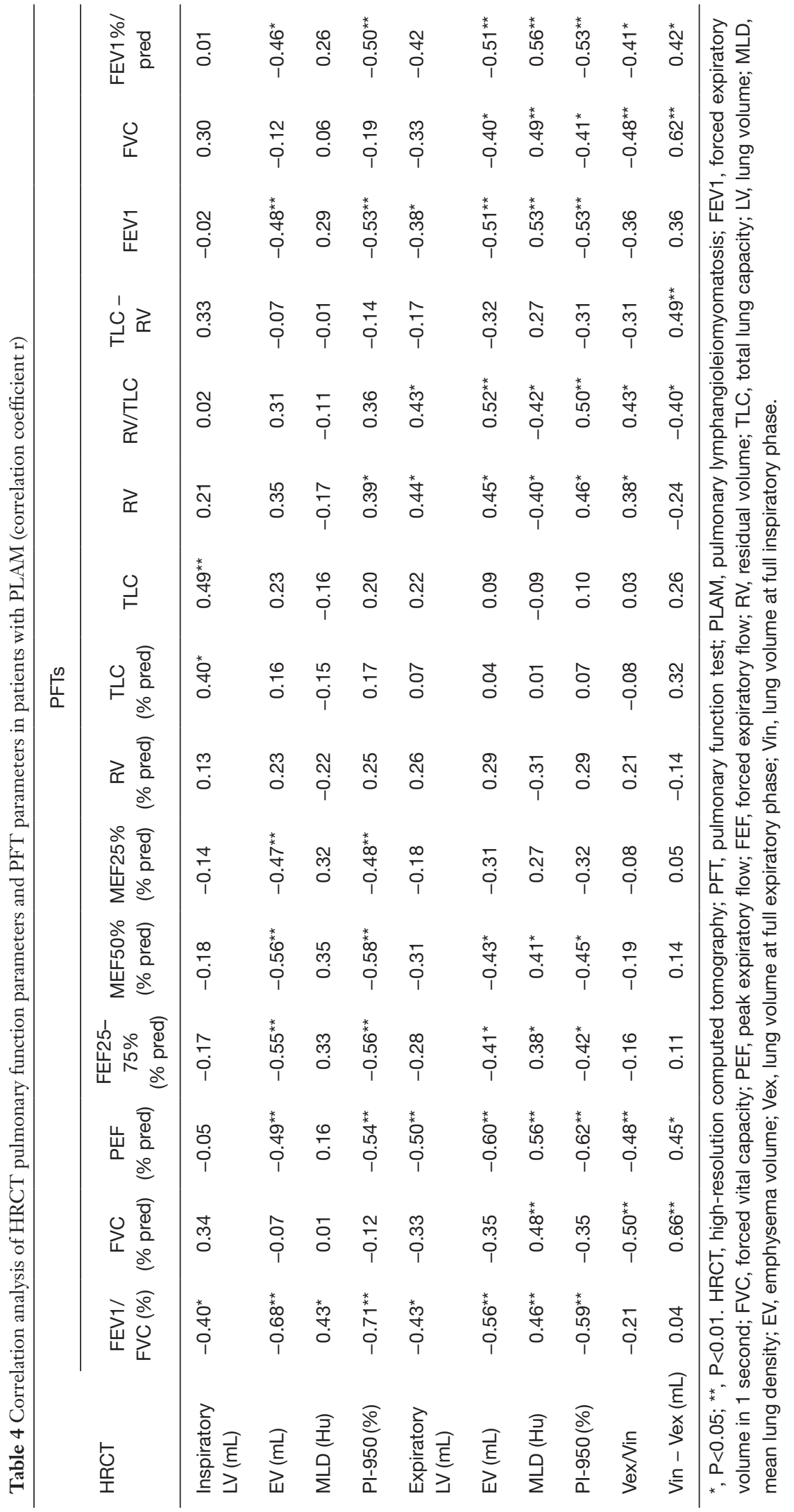



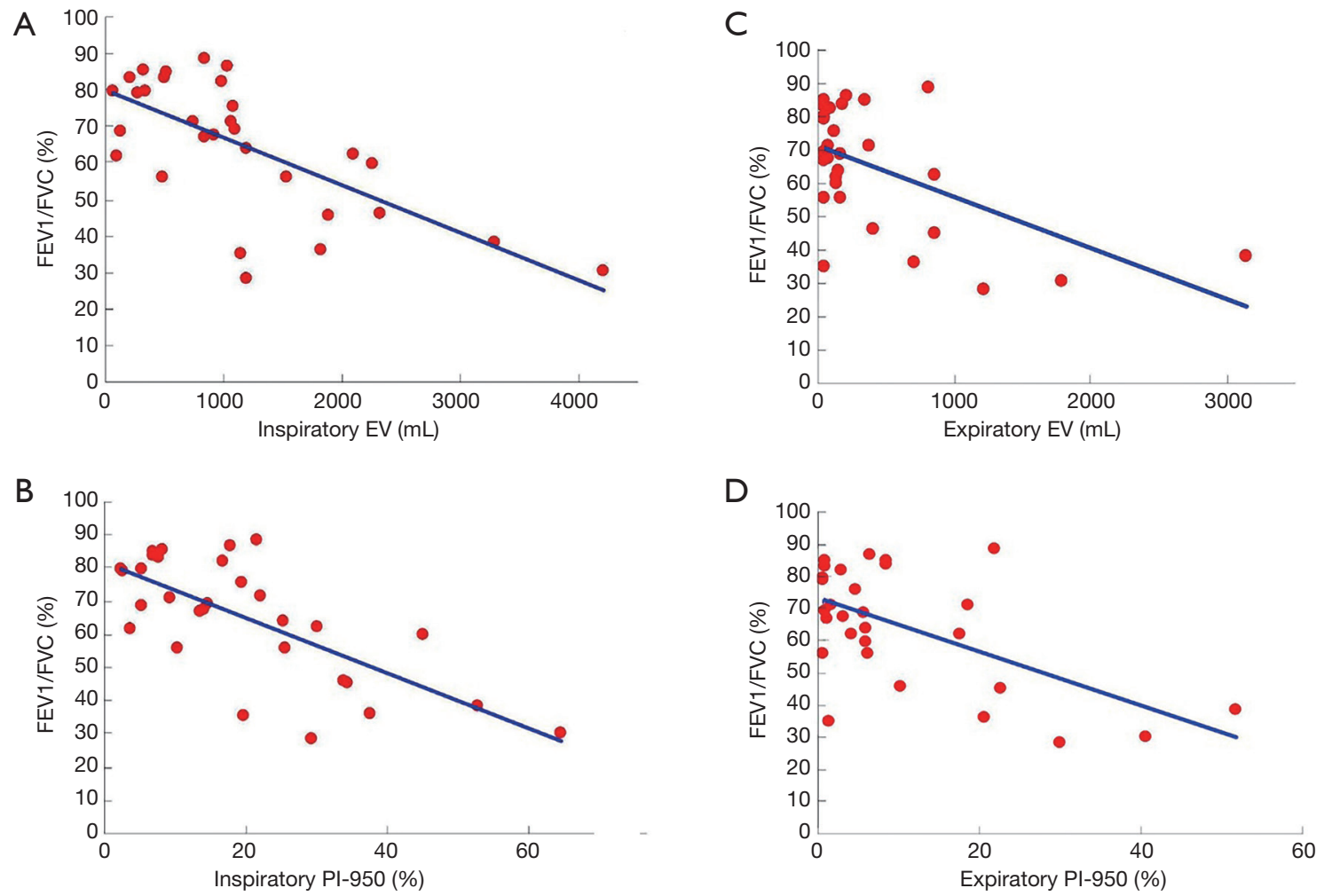

Figure 1 The relationship between FEV1/FVC(\%) and EV, PI-950(\%). (A) Inspiratory EV was negatively correlated with FEV1/FVC (\%) $(\mathrm{r}=-0.68, \mathrm{P}<0.01)$; (B) inspiratory PI-950 (\%) was negatively correlated with FEV1/FVC (\%) $(\mathrm{r}=-0.71, \mathrm{P}<0.01)$; (C) expiratory EV was negatively correlated with FEV1/FVC (\%) ( $\mathrm{r}=-0.56, \mathrm{P}<0.01)$; (D) expiratory PI-950 (\%) was negatively correlated with FEV1/FVC (\%) $(\mathrm{r}=-0.59, \mathrm{P}<0.01)$. EV, emphysema volume; FEV1/FVC (\%), forced expiratory volume in 1 second/forced vital capacity.

Table 5 Statistical results for routine pulmonary function parameters before and after treatment in patients with PLAM

\begin{tabular}{lccc}
\hline PFTs & Before $(n=21)$ & After $(n=21)$ & P value \\
\hline TLC $(L)$ & $4.24 \pm 0.96$ & $4.19 \pm 0.64$ & 0.422 \\
RV $(L)$ & $1.61 \pm 0.62$ & $1.63 \pm 0.53$ & 0.456 \\
FEV1 & $1.62 \pm 0.73$ & $1.53 \pm 0.65$ & 0.338 \\
FVC & $2.52 \pm 0.75$ & $2.50 \pm 0.63$ & 0.463 \\
FVC\% & $0.63 \pm 0.20$ & $0.60 \pm 0.21$ & 0.319 \\
FEV1\%/pred & $58.71 \pm 26.58$ & $57.92 \pm 24.64$ & 0.461 \\
\hline
\end{tabular}

PLAM, pulmonary lymphangioleiomyomatosis; PFTs, pulmonary function tests; RV, residual volume; TLC, total lung capacity; FEV1, forced expiratory volume in 1 second; FVC, forced vital capacity.

The expiratory mean $L V$ was less than that measured before treatment.

\section{Discussion}

PLAM mainly reveals itself through diffuse cystic changes throughout the lungs on CT as well as destruction of the lung structure that leads to the deterioration of pulmonary function. Quantitative HRCT-derived lung function evaluates the extent of air trapping in the lungs as measured by the LV, EV, MLD, and pixel index. Quantitative HRCT showed that the LVs of PLAM patients were lower than 
Table 6 HRCT pulmonary function parameters before and after treatment in patients with PLAM

\begin{tabular}{|c|c|c|c|}
\hline HRCT & Before $(n=21)$ & After $(n=21)$ & $P$ value \\
\hline $\mathrm{LV}(\mathrm{mL})$ & $3,605.11 \pm 922.82$ & $3,947.97 \pm 979.16$ & 0.048 \\
\hline $\mathrm{EV}(\mathrm{mL})$ & $1,130.06 \pm 1,047.55$ & $1,085.35 \pm 1,037.21$ & 0.445 \\
\hline $\operatorname{MLD}(\mathrm{Hu})$ & $-862.57 \pm 45.33$ & $-860.89 \pm 40.86$ & 0.450 \\
\hline \multicolumn{4}{|l|}{ Expiratory } \\
\hline $\mathrm{LV}(\mathrm{mL})$ & $2,413.90 \pm 1,029.54$ & $1,907.45 \pm 935.10$ & 0.032 \\
\hline $\mathrm{EV}(\mathrm{mL})$ & $419.54 \pm 883.00$ & $394.62 \pm 759.04$ & 0.461 \\
\hline MLD (Hu) & $-760.58 \pm 78.03$ & $-768.84 \pm 73.44$ & 0.363 \\
\hline EVin - EVex (mL) & $1,534.07 \pm 893.69$ & $1,697.67 \pm 1,068.20$ & 0.297 \\
\hline
\end{tabular}

HRCT, high-resolution computed tomography; PLAM, pulmonary lymphangioleiomyomatosis; LV, lung volume; MLD, mean lung density; EV, emphysema volume; EVex, emphysema volume at full expiratory phase; EVin, emphysema volume at full inspiratory phase.

the LVs of the control group in the inspiratory phase. This finding is different from the results of previous studies for other emphysematous diseases (7-9). Whereas chronic obstructive pulmonary disease (COPD) patients have mainly been characterized as having enlarged LVs, the LVs of PLAM patients were not significantly enlarged. This contrast reveals that although patients with PLAM have cystic changes, these changes did not result in significant variations in the LV. Prior studies have compared PLAM patients with COPD patients and found that under the same pulmonary function measured by PFTs, quantitative HRCT showed that the areas of parenchymal destruction in PLAM patients were relatively less severe than those of COPD patients and that the percentage of pulmonary small blood vessels and mean area of the lung parenchyma were higher in PLAM patients than in COPD patients (10). Our study suggests that quantitative HRCT can be used to differentiate between the two diseases.

There was a slight but not significant decrease in inspiratory MLD in PLAM patients compared to that in the control group, while the expiratory MLD of the PLAM patients was significantly lower than that of the control group. This finding may be explained by the fact that during the inspiratory phase, healthy patients inhale a large amount of gas into the alveoli, which would have a similar CT density as the structurally damaged lungs in PLAM patients. In the expiratory phase, air trapping within PLAM patients' lungs results in significantly decreased overall lung density compared to healthy subjects.

Currently, PFT remains the gold standard clinical pulmonary function assessment. In this study, we correlated standard PFT parameters with HRCT-derived metrics of lung function. From the results, among the quantitative HRCT parameters of lung function in patients with PLAM, during both the inspiratory and expiratory phases, PI-950 had good positive correlations with FEV1/FVC (\%), PEF (\% pred), FEF $25-75 \%$ (\% pred), and MEF50 (\% pred), and the inspiratory PI-950 had a good negative correlation with FEV1/FVC (\%) at -0.71 . The correlation coefficient between PI-950 and FEV1/FVC (\%) during the expiratory phase was -0.59 , indicating that PI-950 had a good correlation with multiple standard PFT parameters. Our results are similar to those of previous HRCT-derived lung function studies focused on PLAM. According to Ando et al. (10), by selecting $-960 \mathrm{HU}$ as the trigger threshold for the low attenuation region, the percentage of the low attenuation region and FEV1/FVC (\%) showed a correlation coefficient of -0.78 . Kauczor et al. (11) reported that in patients with asthma and COPD, PI-950 had good correlations with FEV1/FVC (\%), PEF (\% pred), and FEF25-75\% (\% pred). The results of that study showed that quantitative HRCT lung function parameters can 
reflect changes in lung function to some extent, i.e., the higher the proportion of lesions below $-950 \mathrm{HU}$, the lower the FEV1/FVC (\%), PEF (\% pred), FEF25-75\% (\% pred), and MEF50 (\% pred), indicating that airflow limitations were significant. These results not only indicated the severity of the cystic lesions in the lungs but also indicated that some small airway lesions should also be combined in PLAM.

In terms of $L V$, our results showed that multiple parameters of HRCT-derived lung function were poorly correlated with RV (\% pred) and TLC (\% pred) during both the inspiratory and expiratory phases, whereby LV showed good correlations with TLC and RV, similar to the results of previous studies $(12,13)$. The poor correlation may suggest the limited applicability of the predicted LV in PLAM patients. These factors require further analysis and study in future works. We found that EV correlated well with multiple clinical indicators, e.g., the correlation coefficient between $\mathrm{EV}$ and FEB1/FVC (\%) was -0.68 , which was compatible with the results of previous emphysema studies by other researchers (14-16). Therefore, it can be concluded that quantitative HRCT metrics are sensitive to emphysematous changes.

Therapeutic response assessment is an important consideration for both clinicians and patients. For pulmonary diseases, the efficacy of treatment is reflected not only in the improvement of patient self-assessments but also in the evaluation of lung function. In recent years, due to the use of new drugs such as the immunosuppressive agent sirolimus, most PLAM patients have achieved improved outcomes $(4,17)$ and prolonged overall survival. To manage these patients, it is very important to regularly assess lung function. At present, although PFT is the gold standard for this purpose, some patients are not suitable for these lung function tests because of the need for repeated inhalation and expiration, which can result in adverse conditions, such as pneumothorax. Thus, we compared treatment response assessments for lung function using standard PFT and HRCT-derived metrics. In contrast to prior reports $(18,19)$, we did not observe significant differences in the PFT parameters before and after treatment of the 21 patients followed in this study. Interestingly, quantitative HRCT analysis at similar timepoints showed significant differences in LV. After treatment, the inspiratory LV increased and the expiratory LV decreased, suggesting that lung compliance improved. However, the EV and PI-950, which mainly show changes in the cystic cavity, were not significantly different before and after treatment. Moreover, the EV and PI-950 decreased after treatment, indicating that the volumes of the cysts in the lungs possibly decreased after treatment. This last observation may provide clinicians with an indicator to evaluate treatment efficacy. Future studies should be performed to confirm this finding in larger patient cohorts. Due to the strong compensatory capacity of the lungs, many patients who have significant lung structural damage, as observed on HRCT scans, do not manifest obvious functional deficits on their PFTs (20).

Although this is relatively less harmful than the clinical value of CT, it should not be ignored. All patients in the study underwent biphasic respiratory scans, and some patients were followed up for 3-6 months intervals. These patients may receive more radiation doses than normal during scans, but they are all within acceptable limits. The study assessed lung function during the inspiratory and expiratory phases showing no significant differences in the correlation between PI-950 and clinical parameters in both phases, suggesting that a single-phase scan may be sufficient for functional assessments to reduce the doses the patients receive.

\section{Conclusions}

Overall, this study revealed that HRCT can provide functional parameters that show good correlation with those obtained with gold standard PFTs, in addition to structural information. Furthermore, HRCT-derived parameters may potentially be more sensitive than PFT parameters during the early phase of treatment response. Therefore, quantitative HRCT is a promising tool for the early diagnosis and treatment assessment of lung function in PLAM patients.

\section{Acknowledgments}

Funding: The work of HC was supported by the following sources: (I) Project of Natural Science Foundation of Guangdong Province (2019A1515011382); (II) The Postgraduate Education Innovation Planning Project of Guangdong Province, China (2018JGXM83); (III) The Science and Technology Planning Project of Guangzhou, Guangdong Province, China (201904010130); (IV) 2018 Achievement Transformation and cultivation Project of the first affiliated Hospital of Guangzhou Medical University (ZH201806); (V) Laboratory Opening Project for College students in 2019 (C19501504). The work of ZWN was supported by the Characteristic Innovation Projects of 
Colleges and Universities in Guangdong Province, China (2018KTSCX188).

\section{Footnote}

Data Sharing Statement: Available at http://dx.doi. org/10.21037/jtd-20-1104

Conflicts of Interest: All authors have completed the ICMJE uniform disclosure form (available at http://dx.doi. org/10.21037/jtd-20-1104). The authors have no conflicts of interest to declare.

Ethical Statement: The authors are accountable for all aspects of the work in ensuring that questions related to the accuracy or integrity of any part of the work are appropriately investigated and resolved. The study was conducted in accordance with the Declaration of Helsinki (as revised in 2013). The study was approved by the institutional ethical committee of the First Affiliated Hospital of Guangzhou Medical University (Medical research ethical review 2019, No. K47), with informed consent waived due to the retrospective nature of the study.

Open Access Statement: This is an Open Access article distributed in accordance with the Creative Commons Attribution-NonCommercial-NoDerivs 4.0 International License (CC BY-NC-ND 4.0), which permits the noncommercial replication and distribution of the article with the strict proviso that no changes or edits are made and the original work is properly cited (including links to both the formal publication through the relevant DOI and the license). See: https://creativecommons.org/licenses/by-nc-nd/4.0/.

\section{References}

1. Avila NA, Andrew J, Antoinette R. Sporadic Lymphangioleiomyomatosis and Tuberous Sclerosis Complex with Lymphangioleiomyomatosis: Comparison of CT Features. Radiology 2007;242:277-85.

2. Cudzilo CJ, Szczesniak RD, Brody AS, et al. Lymphangioleiomyomatosis screening in women with tuberous sclerosis. Chest 2013;144:578-85.

3. Baldi BG, Araujo MS, Freitas CS, et al. Evaluation of the extent of pulmonary cysts and their association with functional variables and serum markers in lymphangioleiomyomatosis (LAM). Lung 2014;192:967-74.

4. McCormack FX, Inoue Y, Moss J, et al. Efficacy and safety of sirolimus in lymphangioleiomyomatosis. N Engl J Med 2011;364:1595-606.

5. Young L, Lee HS, Inoue Y, et al. Serum VEGF-D a concentration as a biomarker of lymphangioleiomyomatosis severity and treatment response: a prospective analysis of the Multicenter International Lymphangioleiomyomatosis Efficacy of Sirolimus (MILES) trial. Lancet Respir Med 2013;1:445-52.

6. Northrup H, Krueger DA. International Tuberous Sclerosis Complex Consensus Group. Tuberous sclerosis complex diagnostic criteria update: recommendations of the 2012 international Tuberous Sclerosis Complex Consensus Conference. Pediatr Neurol 2013;49:243-54.

7. Matsuoka S, Kurihara Y, Yagihashi K, et al.

Morphological progression of emphysema on thin-section $\mathrm{CT}$ : analysis of longitudinal change in the number and size of low-attenuation clusters. J Comput Assist Tomogr 2006;30:669-74.

8. Vikgren J, Friman O, Borga M, et al. Detection of mild emphysema by computed tomography density measurements. Acta Radiol 2005;46:237-45.

9. Bakker ME, Stolk J, Putter H, et al. Variability in densitometric assessment of pulmonary emphysema with computed tomography. Invest Radiol 2005;40:777-83.

10. Ando K, Tobino K, Kurihara M, et al. Quantitative $\mathrm{CT}$ analysis of small pulmonary vessels in lymphangioleiomyomatosis. Eur J Radiol 2012;81:3925-30.

11. Kauczor HU, Wielputz MO, Owsijewitsch M, et al. Computed tomographic imaging of the airways in COPD and asthma. J Thorac Imaging 2011;26:290-300.

12. Crausman RS, Lynch DA, Mortenson RL, et al. Quantitative CT predicts the severity of physiologic dysfunction in patients with lymphangioleiomyomatosis. Chest 1996;109:131-7.

13. Avila NA, Kelly JA, Dwyer AJ, et al. Lymphangioleiomyomatosis: correlation of qualitative and quantitative thin-section CT with pulmonary function tests and assessment of dependence on pleurodesis. Radiology 2002;223:189-97.

14. Nishino M, Washko GR, Hatabu H. Volumetric Expiratory HRCT of the Lung: Clinical Applications. Radiol Clin N Am 2010;48:177-83.

15. Tashkin DP. Relationship between quantitative radiographic assessments of interstitial lung disease and physiological and clinical features of systemic sclerosis. Ann Rheum Dis 2016;75:374-81.

16. Salaffi F, Carotti M, Di Donato E, et al. Computer-Aided Tomographic Analysis of Interstitial Lung Disease (ILD) 
in Patients with Systemic Sclerosis (SSc). Correlation with Pulmonary Physiologic Tests and Patient-Centred Measures of Perceived Dyspnea and Functional Disability. PLoS One 2016;11:e0149240.

17. Bissler JJ, McCormack FX, Young LR, et al. Sirolimus for angiomyolipoma in tuberous sclerosis complex or lymphangioleiomyomatos. N Engl J Med 2008;358:140-51.

18. Kim HJ, Tashkin DP, Gjertson DW, et al. Transitions to different patterns of interstitial lung disease in

Cite this article as: $\mathrm{Ni} \mathrm{Z,} \mathrm{Ng} \mathrm{TSC,} \mathrm{Liu} \mathrm{J,} \mathrm{Huang} \mathrm{S,} \mathrm{Li} \mathrm{X,} \mathrm{Xu}$ $\mathrm{X}$, Chen H. Quantitative assessment of pulmonary function in lymphangioleiomyomatosis patients using high-resolution computed tomography and pulmonary function tests. J Thorac Dis 2020;12(11):6466-6475. doi: 10.21037/jtd-20-1104 scleroderma with and without treatment. Ann Rheum Dis 2016;75:1367-71.

19. Kloth C, Maximilian TW, Preibsch H, et al. Quantitative chest CT analysis in patients with systemic sclerosis before and after autologous stem cell transplantation: comparison of results with those of pulmonary function tests and clinical tests. Rheumatology (Oxford) 2016;5 5:1763-70

20. Trotman-Dickenson B. Cystic lung disease: achieving a radiologic diagnosis. Eur J Radiol 2014;83:39-46. 